\title{
What's Going On with My CryoEM/CryoFIB-SEM Sample, and How Might I Improve It?
}

\author{
Alex Noble, Clint Potter and Bridget Carragher
}

New York Structural Biology Center, New York, New York, United States

Cryo-electron microscopy (cryoEM) opens up the spectrum of in-situ and native biological structure determination to a range of specimen from purified protein samples to cellular/tissue environments. However, several challenges often hamper efforts to prepare optimal or even useful cryoEM grids for TEM collection. Here I will discuss the most common potential issues with both single particle cryoEM and cryo-FIB/SEM sample/grid preparation. We will explore potential practical solutions, with the general aim of helping progress your cryoEM structure-determination project and your knowledge of the issues.

First I will introduce you to the most common potential issues in single particle cryoEM, namely air-water interface adsorption, potential denaturation, and induced preferred orientations. Most purified samples prepared on conventional cryoEM grids adsorb to the air-water interface. We will explore food science literature to understand how denaturation may affect protein structure. We will then explore several published methods for ameliorating air-water interface issues, adjusting sample concentration on the grid, adsorbing proteins to specialized grid substrates, and reducing preferred orientation issues by optimizing collection strategies. Finally, we will look into potential upcoming methods for preparing samples on grids and addressing these issues, including a developmental Spotiton method

Finally, I will provide an introduction to in-situ sample preparation for typical cell specimen and for specimen that require larger volumes than the thin film created for conventional single particle cryoEM. We will briefly introduce cryo-FIB/SEM, discussing several potential issues and solutions, and we will end at a technique called the 'waffle method' for sample preparation that addresses cellular preferred orientation. We will explore how the waffle method solves not only preferred orientation, vitreousness, and sample concentration, but also may uniquely provide a way to study filaments in all orientations and single particle samples truly in bulk.

\section{References}

Carragher, B., et al. (2019). Current outcomes when optimizing 'standard' sample preparation for singleparticle cryo-EM. J. Microsc. 276, 39-45.

Chen, J., Noble, A.J., et al. (2019). Eliminating effects of particle adsorption to the air/water interface in single-particle cryo-electron microscopy: Bacterial RNA polymerase and CHAPSO. J. Struct. Biol. X 1, 100005 .

D'Imprima, E., et al. (2019). Protein denaturation at the air-water interface and how to prevent it. ELife 8, e42747.

Glaeser, R.M. (2017). Proteins, Interfaces, and Cryo-EM Grids. Curr. Opin. Colloid Interface Sci.

Han, Y., et al. (2020). High-yield monolayer graphene grids for near-atomic resolution cryoelectron microscopy. Proc. Natl. Acad. Sci. 117, 1009-1014.

Lyumkis, D. (2019). Challenges and Opportunities in Cryo-EM Single-Particle Analysis. J. Biol. Chem. jbc.REV118.005602.

Noble, A.J., et al. (2018a). Routine single particle CryoEM sample and grid characterization by tomography. ELife 7, e34257. 
Noble, A.J., Wei, H., Dandey, V.P., et al. (2018b). Reducing effects of particle adsorption to the air-water interface in cryo-EM. Nat. Methods 15, 793-795.

Snijder, J., et al. (2017). Vitrification after multiple rounds of sample application and blotting improves particle density on cryo-electron microscopy grids. J. Struct. Biol. 198, 38-42.

Tan, Y.Z., et al. (2017). Addressing preferred specimen orientation in single-particle cryo-EM through tilting. Nat. Methods 14, 793-796.

Villa, E., et al. (2013). Opening windows into the cell: focused-ion-beam milling for cryo-electron tomography. Curr. Opin. Struct. Biol. 23, 771-777. 